\title{
Surface Enhanced Raman Spectroscopy Using a Single Mode Nanophotonic-Plasmonic Platform
}

\author{
Frédéric Peyskens, ${ }^{*, \dagger}$ Ashim Dhakal,${ }^{\dagger}$ Pol Van Dorpe,${ }^{\ddagger}$ Nicolas Le \\ Thomas, $^{\dagger}$ and Roel Baets ${ }^{\dagger}$
}

Photonics Research Group, INTEC-department, Ghent University-imec; Center for Nano-and BioPhotonics, Ghent University, Sint-Pietersnieuwstraat 41, 9000 Ghent, Belgium, and imec, Kapeldreef 75, 3001 Heverlee, Belgium; Department of Physics, KULeuven, Celestijnenlaan 200D, 3001 Leuven, Belgium

E-mail: fpeysken@intec.ugent.be

\begin{abstract}
We demonstrate for the first time the generation of Surface Enhanced Raman Spectroscopy (SERS) signals from integrated bowtie antennas, excited and collected by the fundamental TE-mode of a single mode silicon nitride waveguide. Due to the integrated nature of this particular single mode SERS-probe one can rigorously quantify the complete enhancement process. The Stokes power, generated by a 4-Nitrothiophenol-coated antenna and collected into the fundamental TE-mode, exhibits an $8 \times 10^{6}$ enhancement compared to the free-space Raman scattering of a 4-Nitrothiophenol molecule.

${ }^{*}$ To whom correspondence should be addressed

${ }^{\dagger}$ Photonics Research Group, INTEC-department, Ghent University-imec; Center for Nano-and BioPhotonics, Ghent University, Sint-Pietersnieuwstraat 41, 9000 Ghent, Belgium

${ }^{\ddagger}$ imec, Kapeldreef 75, 3001 Heverlee, Belgium; Department of Physics, KULeuven, Celestijnenlaan 200D,
\end{abstract} 3001 Leuven, Belgium 
Furthermore we present an analytical model which identifies the relevant design parameters and figure of merit for this new SERS-platform. An excellent correspondence is obtained between the theoretically predicted and experimentally observed absolute Raman power. This work paves the way towards a new class of fully integrated labon-a-chip systems where the single mode SERS-probe can be combined with other photonic, fluidic or biological functionalities.

KEYWORDS: Surface Enhanced Raman Spectroscopy, single mode waveguide, photonic integrated circuits, bowtie nanoantenna, plasmonic modeling 
Surface Enhanced Raman Spectroscopy (SERS) is a promising technique for enhancing inherently weak Raman signals by introducing a plasmonic nanostructure in the vicinity of the analyte under study. ${ }^{1-12}$ SERS spectra have mainly been generated using simple plasmonic substrates or colloidal solutions of metallic nanoparticles, whereby the signal is excited and collected by a conventional microscopy system. Recently, photonic integrated circuits (PICs) emerged as a promising alternative to standard confocal microscopy to probe spontaneous Raman spectra. ${ }^{13}$ The fundamental building block of such circuits is a single mode waveguide in which the pump and Stokes light is guided in a high-index core material surrounded by lower-index cladding materials. While researchers also started integrating nanoplasmonic antennas on top of such waveguides, ${ }^{14-20}$ PICs have only been used to probe SERS signals from external, non-integrated, metallic nanoparticles. ${ }^{21-23}$ Such an approach however suffers from the same drawbacks of conventional SERS, in the sense that it is poor in terms of quantitative results owing to the large uncertainty on the Raman enhancement and coupling between the excitation beam and the metallic nanoparticles. In order to obtain quantitative SERS spectra a complete control of the plasmonic enhancement and coupling with the underlying waveguide is necessary.

Here we present a new class of SERS-probes which overcomes these issues. We demonstrate for the first time the generation of SERS signals from integrated bowtie nanoantennas, excited and collected by a single mode silicon nitride waveguide. Due to the fully integrated nature of the developed single mode SERS-probe it is possible to rigorously quantify the complete enhancement and coupling process. We show that the SERS spectra can be attributed to a genuine plasmon resonance effect and experimentally determine the enhanced Stokes power, generated by a single nanoantenna, which is coupled into the fundamental TE-mode. Furthermore an analytical model identifying the relevant design parameters and figure of merit for waveguide-based SERS is developed as well. The theoretically predicted absolute Raman power perfectly corresponds with the experimentally observed power coupled into the fundamental TE-mode. Finally we discuss the impact of the shot noise resulting 
from the inherent silicon nitride Raman background and outline the interplay between signal optimization and noise reduction in order to achieve the optimal absolute detection limit.

A schematic of the device under study is shown in Figure 1(a). The fundamental TE-mode of a silicon nitride (SiN) rib waveguide excites a periodic array of gold bowtie antennas coated with a 4-Nitrothiophenol (NTP) monolayer. The pump wavelength for all experiments is set to $\lambda_{P}=785 \mathrm{~nm}$ and NTP Stokes light (at $\lambda_{S}$ ) is subsequently collected back into the same waveguide mode. Fabrication details can be found in the Methods section and a description of the measurement setup is outlined in the Supporting Information S1. A scanning electron microscope image of the functionalized waveguide, i.e. a waveguide patterned with plasmonic nanoantennas, is depicted in Figure 1(b). All waveguides have a cross-sectional area of $220 \times 700 \mathrm{~nm}^{2}$. Raman spectra of an uncoated (without NTP) and coated (with NTP) waveguide functionalized with 40 antennas are shown in Figure 1(c). The spectral regions where an NTP Stokes peak is expected $\left(1080,1110,1340 \text { and } 1575 \mathrm{~cm}^{-1}\right)^{24}$ are highlighted by the cyan shaded areas. Before coating no NTP peaks can be distinguished from the inherent SiN background. After coating, four additional peaks appear and coincide with the expected NTP Stokes peaks. This demonstrates that SERS signals from single monolayer coated antennas can be efficiently excited and collected by the same fundamental waveguide mode. The peaks at 1250 and $1518 \mathrm{~cm}^{-1}$ (marked by the black dashed lines) are attributed to interference effects of the Au array which act on the scattered background light, and they are also observed on the extinction curves of the functionalized waveguides (see Supporting Information S2). Hence they do not represent specific Raman lines.

Subsequently the dependence of the SERS signal on the position of the plasmon resonance was investigated to verify that it can be attributed to a resonance effect and not to coincidental surface roughness. To this end, waveguides functionalized with a fixed number of antennas but varying bowtie geometries were considered. The relevant bowtie parameters are its length $L$, gap $\Delta$ and apex angle $\alpha$ (Figure 2(a)). By changing the length, the antenna resonance can be tuned $\left(L_{1}=90 \mathrm{~nm}, L_{2}=115 \mathrm{~nm}\right.$ and $L_{3}=140 \mathrm{~nm}$ for fixed $\alpha=60^{\circ}$ and 
(a)

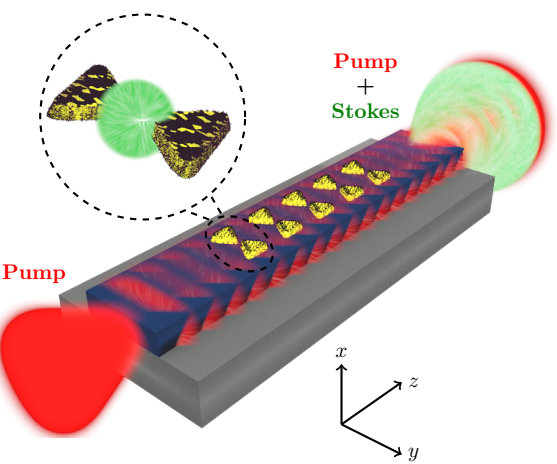

(c)
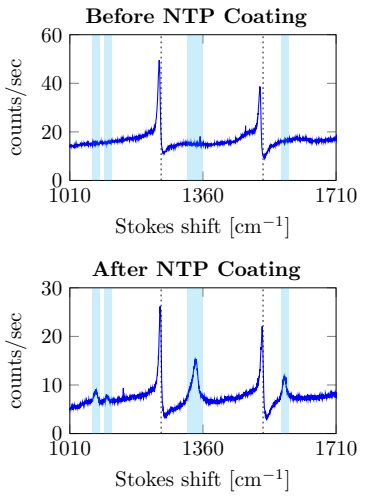

(b)

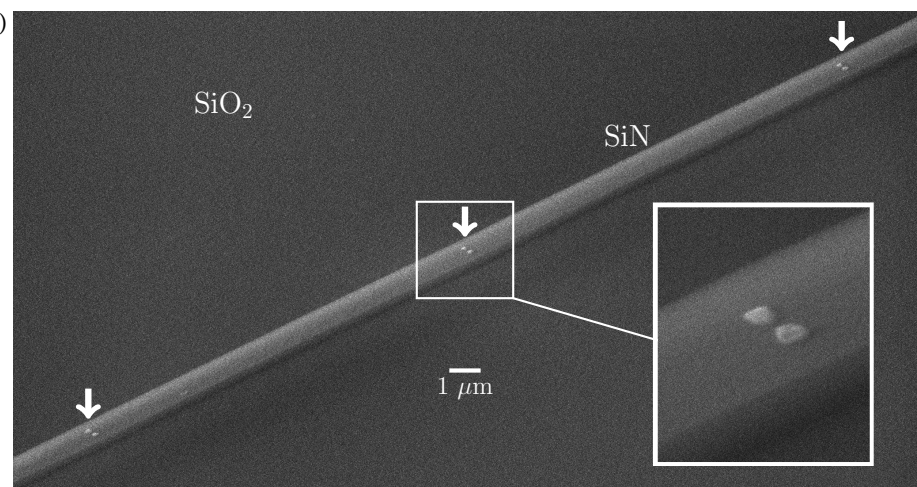

Figure 1: Evanescent excitation and collection of SERS spectra. (a) Schematic of the chip consisting of single mode SiN waveguides (blue) on an $\mathrm{SiO}_{2}$ undercladding (gray), functionalized with an array of gold bowtie antennas (yellow). All antennas are coated with an NTP monolayer (purple dots), evanescently excited by the fundamental TE-mode (red). The NTP Stokes signal (green) is collected by the same mode. (b) Scanning electron microscope image of a functionalized waveguide. The white arrows indicate antenna positions. The inset shows a zoom of a typical antenna. (c) Raman spectra of a waveguide functionalized with 40 antennas. The cyan shaded areas mark the NTP Stokes peaks while the black dashed lines represent peaks attributed to interference effects of the plasmonic array. Before coating, the waveguide already generates a Raman background in itself (top). After coating, NTP peaks emerge (bottom).

\section{Figure 1}


$\Delta=40 \mathrm{~nm}$ ). Extinction spectra are plotted in Figure 2(b) while the corresponding Raman spectra are depicted in Figure 2(c). The Raman spectrum of a reference waveguide without any $\mathrm{Au}$ functionalization is also shown. Even after coating the reference waveguide does not generate NTP peaks, so any Raman signal indeed originates from the antenna region and does not contain contributions from spontaneous Raman scattering along the waveguide. ${ }^{13}$ The $L_{1}$ resonance is detuned from the relevant pump and Stokes region, resulting in a poor Raman spectrum. By increasing the length ( $L_{2}$ and $L_{3}$ bowties) the resonance redshifts and lines up with the pump and Stokes wavelengths. For these bowties the NTP spectrum starts to emerge. The reported SERS spectra can hence be attributed to a plasmon resonance effect such that a stable and reproducible enhancement factor can be associated with them, in contrast to SERS events originating from random surface defects. The increased overlap with the plasmon resonance, and hence extinction, also results in a decreased background.
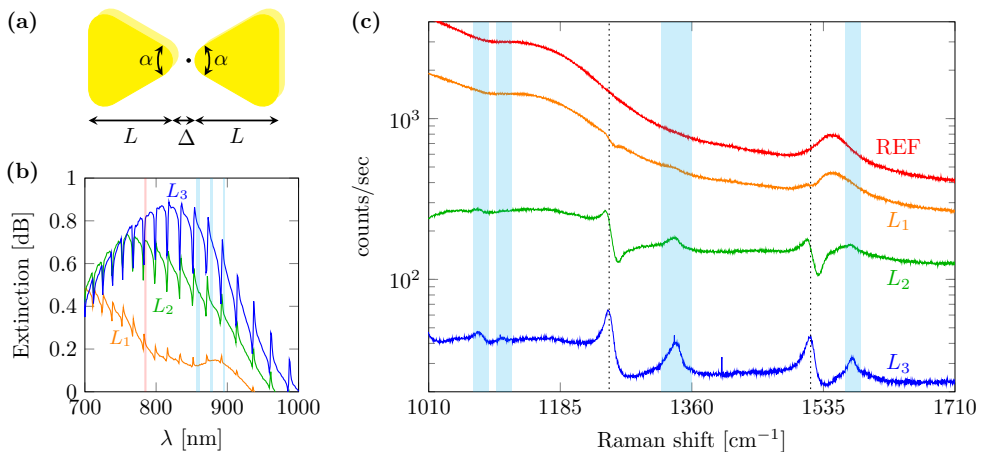

Figure 2: Signal dependence on the plasmon resonance.(a) Geometrical parameters of a bowtie antenna: length $L$, gap $\Delta$ and apex angle $\alpha$. (b) Single antenna extinction spectra for 3 different bowtie antennas with fixed apex angle $\alpha=60^{\circ}$ and gap $\Delta=40 \mathrm{~nm}$ but varying length: $L_{1}=90 \mathrm{~nm}$ (orange), $L_{2}=115 \mathrm{~nm}$ (green), $L_{3}=140 \mathrm{~nm}$ (blue). The red and cyan shaded lines correspond to the pump and Stokes wavelengths respectively. (c) Corresponding Raman spectra of the waveguides functionalized with these 3 bowtie anntenas (orange,green,blue) and Raman spectrum of the reference waveguide (red).

Figure 2

Due to the metal induced loss, there will exist an optimum number $N_{\text {opt }}$ of patterned 
antennas such that the SERS signal is maximized. Such an optimum is investigated in Figure 3 for a fixed bowtie geometry $\left(\alpha=60^{\circ}, L=100 \mathrm{~nm}\right.$ and $\left.\Delta=40 \mathrm{~nm}\right)$ but varying $N$ : $N=10,20,30,40,70$ and $N=0$ which is a reference waveguide. Each waveguide is measured 10 times and the averaged Raman spectra are reported in Figure 3(a). The $N=70$ signal is not shown since it could not be distinguished from the inherent offset signal of the detector. For a given fixed input power, corresponding to roughly $5 \mathrm{~mW}$ guided power, the reference waveguide generates a considerable background signal in the $1340 \mathrm{~cm}^{-1}$ region, where the strongest NTP peak is expected. Functionalizing the waveguide with increasing $N$ reduces this unwanted background due to the attenuation caused by the nanoantennas. In addition the $1340 \mathrm{~cm}^{-1}$ peak starts to emerge when $N$ increases. The smaller peaks at 1080, 1110 and $1575 \mathrm{~cm}^{-1}$ only appear when the background is sufficiently low. A zoom on the dominant $1340 \mathrm{~cm}^{-1}$ peak (cyan dashed line) is shown in Figure 3(b). For clarity the background is locally subtracted. As expected, the signal reaches a maximum value for $10 \leq N \leq 20$ and then decays again with increasing $N$. Apart from signal optimization it is however equally important to reduce the $\mathrm{SiN}$ background in order to resolve the smallest spectral features. Therefore an analytical model is developed to outline the interplay between signal enhancement and background reduction, and to derive the relevant figure of merit for on-chip SERS.

Figure 4(a) shows a schematic longitudinal cross-section of the chip consisting of $N$ antennas spaced with period $\Lambda=10 \mu \mathrm{m}$. Each array is centered on the waveguide with a distance $L_{1} \approx 0.5 \mathrm{~cm}$ to the front and back facet of the chip. The NTP monolayer on each antenna will generate a forward propagating Stokes power $P_{A}\left(\lambda_{P}, \lambda_{S}\right)$ for a given pump power $P_{\text {pump }}$. This single antenna conversion efficiency $P_{A}\left(\lambda_{P}, \lambda_{S}\right)$ is an antenna dependent factor incorporating the integrated field enhancement profile near the metal surface and the molecular density and Raman cross-section. The total transmission loss induced by one antenna at wavelength $\lambda$ is given by $1-e_{\lambda}^{-1}$, whereby $e_{\lambda}$ is the linear antenna extinction. Apart from the intrinsic waveguide losses $\alpha_{w g}$, the pump and Stokes light will hence also be 

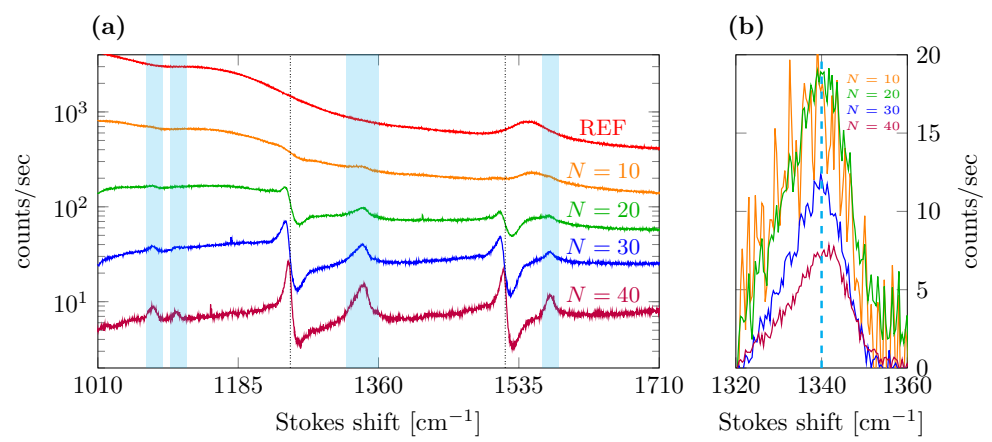

Figure 3: Signal dependence on the number of antennas $N$. (a) Raman spectra of a reference waveguide (REF) and waveguides functionalized with $N=10,20,30,40$ antennas. (b) Zoom on the $1340 \mathrm{~cm}^{-1}$ signal peak. The background is locally subtracted to obtain the pure NTP signal.

Figure 3

attenuated by $e_{P}$ and $e_{S}$ respectively. In the Supporting Information S3 it is then shown that the total Stokes power $P_{\text {tot }}$ generated by an array of $N$ coated antennas is approximately given by

$$
\frac{P_{t o t}}{P_{\text {pump }}} \approx P_{A}\left(\lambda_{P}, \lambda_{S}\right) \mathrm{e}^{-2 \alpha_{w g} L_{1}} e_{S}^{(1-N)}\left(\frac{1-\left(\frac{e_{S}}{e_{P}}\right)^{N}}{1-\left(\frac{e_{S}}{e_{P}}\right)}\right)=F O M\left(N, \lambda_{P}, \lambda_{S}\right) \mathrm{e}^{-2 \alpha_{w g} L_{1}}
$$

The quantity $\operatorname{FOM}\left(N, \lambda_{P}, \lambda_{S}\right)$ contains all necessary parameters to assess the SERS signal strength for a given waveguide geometry and is hence considered to be the relevant figure of merit $(F O M)$ in comparing the performance of integrated antenna arrays. The optimum antenna number $N_{\text {opt }}=\log \left\{\log \left(e_{S}\right) / \log \left(e_{P}\right)\right\} / \log \left(e_{S} / e_{P}\right)$. For the particular bowtie antenna studied in Figure 3, the extinction spectrum $e(\lambda)$ and single antenna conversion efficiency $P_{A}\left(\lambda_{P}, \lambda_{S}\right)$ are numerically evaluated using Lumerical FDTD Solutions (see Methods section). The predicted $N_{\text {opt }}$ for the $1340 \mathrm{~cm}^{-1}$ peak is 10 antennas, using the simulated extinctions $e_{P}=1.14\left(E_{P}=0.58 \mathrm{~dB}\right)$ and $e_{S}=1.08\left(E_{S}=0.34 \mathrm{~dB}\right)$, while $P_{A} \approx 2.35 \times 10^{-15}$. For each $1 \mathrm{~W}$ of pump power the antenna is therefore expected to generate $2.35 \mathrm{fW}$ of guided Stokes power. The Raman enhancement factor is calculated through 
$E F_{R}=\beta\left(\lambda_{P}\right)^{2} \beta\left(\lambda_{S}\right)^{2}$ in which $\beta(\lambda)$ is the local field enhancement (see Methods section). In the center of the gap at $5 \mathrm{~nm}$ from the tip of the antenna (marked by the black dot in Figure $2(\mathrm{a}))$ an $E F_{R} \approx 1.42 \times 10^{4}$ is expected. Apart from the relevant NTP signal, the SiN itself generates a considerable background while the pump beam is propagating along the waveguide. This background signal $P_{b g}$ can be approximated by

$$
\frac{P_{b g}}{P_{p u m p}} \approx P_{B}\left(\lambda_{P}, \lambda_{S}\right) \mathrm{e}^{-2 \alpha_{w g} L_{1}}\left(e_{P}^{-N}+e_{S}^{-N}\right) L_{1}
$$

in which $P_{B}\left(\lambda_{P}, \lambda_{S}\right)$ is a waveguide dependent factor incorporating the specific modal field profile and the SiN molecular density and cross-section (see Supporting Information S4). It is clear that the background increases linearly with the total propagation length $\left(\propto L_{1}\right)$ and decays exponentially with the number of antennas. In order to achieve the highest signal-to-noise ratio (SNR) it is hence important to keep $L_{1}$ as short as possible.

Our analytical model and the associated numerical calculations will now be benchmarked against the spectra from Figure 3 to verify whether the theoretically estimated power values correspond to the experimentally obtained absolute Raman powers. To this end, the NTP signal strength at $1340 \mathrm{~cm}^{-1}$, obtained from Figure $3(\mathrm{~b})$, is analyzed as a function of the antenna number $N$ and compared with the theoretical estimations. Furthermore, the background associated shot noise is also calculated. The results are depicted in Figure 4(b). While the ideal model assumes $N$ identical antennas, the fabricated antennas will show differences among each other resulting in changes of $e_{P}, e_{S}$ and $P_{A}$ from one antenna to the other. A generalized model incorporating potential differences in $e_{P}, e_{S}$ and $P_{A}$ is described in the Supporting Information S5. In order to estimate the uncertainty on these experimental parameters a randomized fit to the generalized model has been applied. A set of normally distributed numbers is generated for each of the three parameters and then plugged into the generalized model to calculate the distribution of signal and shot noise counts, defining an area within which the probable signal (blue area) and noise (red area) 
(a)

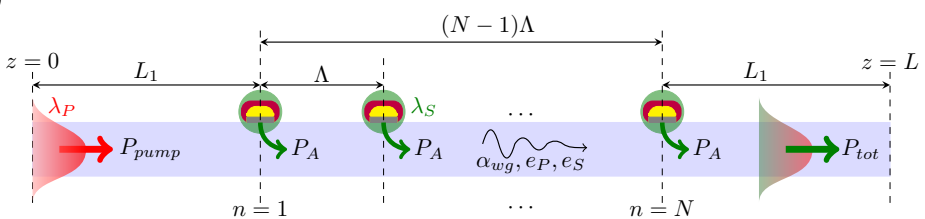

(b)

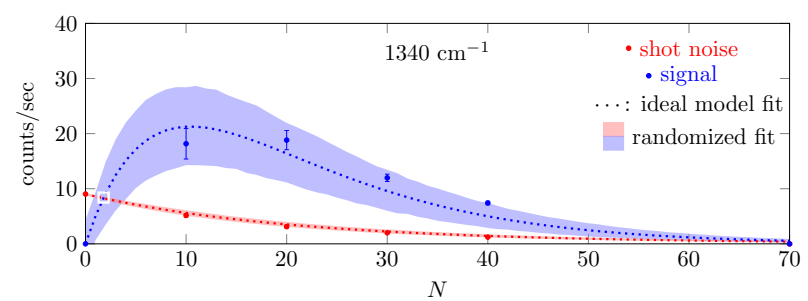

Figure 4: On-chip SERS model and fit to the experimental data. (a) Longitudinal cross-section of the chip. The pump beam, with power $P_{\text {pump }}$ at wavelength $\lambda_{P}$, excites a Stokes signal $\lambda_{S}$ of which the total power at the output facet is $P_{\text {tot }}$. The plasmonic array consists of $N$ antennas with period $\Lambda$ and is separated $L_{1}$ from both the input and output facet of the chip. Each of the antennas generates $P_{A}$ guided Stokes power for a given pump power. Apart from the waveguide losses $\alpha_{w g}$, the pump and Stokes light is attenuated by the pump $e_{P}$ and Stokes $e_{S}$ extinction respectively. (b) Signal (blue circles) and shot noise (red circles) at the $1340 \mathrm{~cm}^{-1}$ peak. The dotted lines represent a constrained fit to the ideal model while the shaded areas represent a randomized fit to estimate the uncertainty on the experimental parameters. The white square denotes the minimum number of antennas $N_{\min }$ required to generate a detectable signal.

\section{Figure 4}


counts are situated. The mean values of these distributions are extracted from an initial constrained fit to the ideal model (dotted lines), while its standard deviations are chosen such that the corresponding signal and shot noise distributions cover all experimental datapoints (red and blue dots). Based on the randomized fit it is possible to estimate the spread on the experimental parameters: $E_{P} \approx 0.49 \pm 0.11 \mathrm{~dB}, E_{S} \approx 0.35 \pm 0.11 \mathrm{~dB}$ and $P_{A}=(2.60 \pm 0.77) \times 10^{-15}$ (theoretically $E_{P}=0.58 \mathrm{~dB}, E_{S}=0.34 \mathrm{~dB}$ and $P_{A}=2.35 \times 10^{-15}$ were predicted). The theoretically predicted parameters are all within the error bars of the experimentally fitted data, which clearly establishes the validity of our model and its ability to provide quantitative predictions of the absolute Raman power coupled into a single mode waveguide. Given this excellent correspondence, we expect the fabricated structures to have a Raman enhancement factor $E F_{R}$ on the order of $1.42 \times 10^{4}$ near the two antenna gap tips. Decreasing the gap size should boost $E F_{R}$ and $P_{A}$ by another two or three orders of magnitude. From the fitting values the optimum antenna number is estimated to be $11 \pm 3$ (compared to 10 theoretically). The single antenna conversion efficiency $P_{A}$ shows that the fabricated antennas produce $(2.60 \pm 0.77) \mathrm{fW}$ of guided Stokes power for each $1 \mathrm{~W}$ of guided pump power. Compared to the free space Raman scattering $P_{0}$ of a single NTP molecule in a bulk air environment $P_{A} \approx 3.94 \times 10^{6} P_{0}$. This includes the excitation and emission enhancement of all molecules in the monolayer as well as the coupling efficiency to the guided mode. Since only half of the Stokes light is carried by the forward propagating mode, the total power coupled into the fundamental TE-mode is therefore $\approx 8 \times 10^{6} P_{0}$.

Our observations also reveal that a minimum number of antennas $N_{\min }$ is required to generate a detectable signal (marked by the white square in Figure 4(b)). If $N<N_{\text {min }}$ then the shot noise still dominates on the signal. It has to be noted however that the relevant signal is generated in a very small region $(N-1) \Lambda$ compared to the overall length $2 L_{1}+(N-1) \Lambda \approx 2 L_{1}$, while the shot noise is mainly attributed to this non-useful length $2 L_{1} \approx 10000 \mu \mathrm{m}$. Practically the antenna section can be made as short as $\approx 10 \mu \mathrm{m}$ for a single antenna. Consequently, designs in which dedicated on-chip splitters are used to 
separate the pump and Stokes light should in principle allow to reduce the background by a factor of $10000 / 10=1000$. These devices are therefore expected to have a $\sqrt{1000} \approx 30$ times higher SNR, pushing towards the $N_{\text {min }}=1$ limit such that signals generated by a single antenna can still be detected. As a result it would become possible to simultaneously probe large areas of analytes $\left(>\lambda^{2}\right)$ and detecting all SERS events originating from different locations by monitoring just a single waveguide output, in contrast to microscopy based systems where one has to serially scan all hotspot locations.

We have fabricated a fully integrated single mode SERS-probe enabling on-chip excitation and emission enhancement in the 700-1000 $\mathrm{nm}$ region and developed an analytical model to outline the relevant design parameters and figure of merit for this new platform. It is shown that the SERS spectra can be attributed to a plasmon resonance effect and that the Stokes power coupled into the fundamental TE-mode perfectly matches with the theoretical predictions extracted from our analytical model. As a result, the suggested platform enables a complete quantitative control on the Raman enhancement and subsequent coupling of the enhanced Stokes light with the underlying waveguide. Furthermore we established, theoretically and experimentally, the existence of an optimal number of antennas to maximize the SERS signal and discussed the impact of the SiN background noise on the absolute detection limit, while providing strategies to simultaneously optimize the signal and mitigate the noise. Due to the low fluorescence, negligible water absorption and availability of high quality and low-cost sources and detectors the 700-1000 nm wavelength region is of particular interest for Raman sensing. ${ }^{25}$ In combination with other on-chip spectral functionalities, such as arrayed waveguide gratings, ${ }^{25}$ the presented SERS-probe is forecasted to allow multiplexed detection of extremely weak Raman signals on a highly dense integrated platform. We also envisage that integrated nanoantennas, similar to the ones reported here, could be used as transducer between quantum dot emitters and the fundamental waveguide mode, potentially enabling applications in on-chip quantum communication and quantum computation. ${ }^{26,27}$ 


\section{Methods}

\section{Fabrication details}

The fabrication consists of a 2-step e-beam lithography process. In the first step the nanoplasmonic antennas are patterned on top of a slab $\mathrm{Si} / \mathrm{SiO}_{2} / \mathrm{SiN}$ wafer using a positive PMMA e-beam resist. After PMMA exposure, the samples are developed in a 1:1 MIBK:IPA solution after which a $2 \mathrm{~nm}$ Ti adhesion layer and $30 \mathrm{~nm}$ Au layer are deposited in a commercial Pfeiffer Spider sputter system. The samples are then immersed in acetone for lift-off. In the second step the waveguides are defined. After metal lift-off a negative ma-N 2403 resist is spun, exposed and developed in ma-D 525. An e-spacer is also spun on top of the ma-N 2403 to avoid charging effects. The developed samples are then etched with an ICP plasma $\left(\mathrm{C}_{4} \mathrm{~F}_{8} / \mathrm{SF}_{6}\right.$ mixture $)$ in a commercial Oxford Plasmalab system. After resist strip and cleaning, the samples are immersed overnight in a $1 \mathrm{mM} \mathrm{NTP:EtOH} \mathrm{solution} \mathrm{and} \mathrm{subsequently}$ rinsed with pure ethanol to remove the residual NTP. A self-assembled monolayer of NTP is assumed to form on the Au surface through a Au-S bond. ${ }^{24}$

\section{Numerical Simulations}

Numerical simulations were performed with Lumerical FDTD Solutions. We used a refractive index of $n_{\text {rib }}=1.9$ for the SiN rib (with width $w_{\text {rib }}=700 \mathrm{~nm}$ and height $h_{\text {rib }}=220 \mathrm{~nm}$ ), $n_{\text {uclad }}=1.45$ for the $\mathrm{SiO}_{2}$ undercladding and $n_{\text {tclad }}=1$ for the top cladding (air). The Si substrate was not taken into account since the real oxide cladding is thick enough to avoid substantial power leakage to the Si such that the numerical results faithfully represent the actual experimental conditions. A thin native oxide layer $\left(t_{n o x}=2 \mathrm{~nm}\right)$ between the $\mathrm{SiN}$ and the Ti has also been incorporated. ${ }^{17}$ The metal stack thicknesses are fixed to $t_{T i}=2$ $\mathrm{nm}$ and $t_{A u}=30 \mathrm{~nm}$ and a built-in refractive index model for Au (Johnson and Christy ${ }^{28}$ ) and $\mathrm{Ti}\left(\mathrm{CRC}^{29}\right)$ is used. An additional surface layer with thickness $t_{N T P}=1 \mathrm{~nm}$ and index $n_{N T P}=3$ is used to model the NTP monolayer. The antenna region (including the Ti 
adhesion layer and the NTP monolayer) is meshed with a uniform mesh of $0.5 \mathrm{~nm}$ in the plane of the antenna ( $y z$-plane) and $2 \mathrm{~nm}$ in the $x$-direction. A mesh refinement to $1 \mathrm{~nm}$ is applied in regions where the thicknesses in the $x$-direction are $\leq 2 \mathrm{~nm}$. The estimated surface area of an NTP molecule is $0.18 \mathrm{~nm}^{2}$, so the surface density is then $\rho_{s}=5.56 \times 10^{18}$ molecules $/ \mathrm{m}^{2} .{ }^{24}$ The Raman cross section is $\sigma \approx 0.358 \times 10^{-30} \mathrm{~cm}^{2} / \mathrm{sr}$, which was obtained by applying the $\lambda_{S}^{-4}$ scaling to the original data of the $1340 \mathrm{~cm}^{-1}$ line. ${ }^{30}$ Single antenna extinction spectra $E(\lambda)$ (in $\mathrm{dB}$ ) are calculated through $E(\lambda)=T_{\text {ref }}(\lambda)-T_{\text {ant }}(\lambda)$ in which $T_{r e f}(\lambda)$ is the power transmission (in $\mathrm{dB}$ ) through the reference waveguide and $T_{\text {ant }}(\lambda)$ the power transmission (in $\mathrm{dB}$ ) of a waveguide functionalized with one antenna. Linear extinction spectra $e(\lambda) \triangleq e_{\lambda}$ are then given by $e(\lambda)=10^{E(\lambda) / 10}$. A field and index profile monitor are used to extract the local field $|\mathbf{E}(\mathbf{r}, \lambda)|$ and index $n(\mathbf{r})$ around the antenna. The single antenna conversion efficiency

$$
P_{A}\left(\lambda_{P}, \lambda_{S}\right)=\frac{\rho_{s} \sigma}{2 t_{N T P}} \frac{\iiint_{V_{m}} n_{g}\left(\lambda_{P}\right) n_{g}\left(\lambda_{S}\right) \lambda_{S}^{2}\left|\mathbf{E}\left(\mathbf{r}, \lambda_{P}\right)\right|^{2}\left|\mathbf{E}\left(\mathbf{r}, \lambda_{S}\right)\right|^{2} d \mathbf{r}}{\left(\iint(\mathbf{r})^{2}\left|\mathbf{E}^{m}\left(\mathbf{r}, \lambda_{P}\right)\right|^{2} d \mathbf{r}\right)\left(\iint n(\mathbf{r})^{2}\left|\mathbf{E}^{m}\left(\mathbf{r}, \lambda_{S}\right)\right|^{2} d \mathbf{r}\right)}
$$

is calculated by integrating the local fields over the effective monolayer volume $V_{m}$ in which the index satisfies $\left.n(\mathbf{r})\right|_{\mathbf{r} \in V_{m}}=n_{N T P}$ (see Supporting Information S3). The group index of the waveguide mode is $n_{g}(\lambda)$. The denominator is calculated using the modal fields $\mathbf{E}^{m}(\mathbf{r}, \lambda)$ of a non-functionalized reference waveguide and the local field enhancement is given by the ratio of the local and modal electric fields: $\beta(\mathbf{r}, \lambda)=\frac{|\mathbf{E}(\mathbf{r}, \lambda)|}{\left|\mathbf{E}^{m}(\mathbf{r}, \lambda)\right|}$. At a certain position, the Raman enhancement factor $E F_{R}$ is calculated as $E F_{R}=\beta\left(\lambda_{P}\right)^{2} \beta\left(\lambda_{S}\right)^{2}$. Numerically calculated values are mentioned in the main text.

\section{Additional information}

Supporting Information is available. 


\section{References}

(1) Anker, J. N.; Hall, W. P.; Lyandres, O.; Shah, N. C.; Zhao, J.; Van Duyne, R.P. Biosensing with plasmonic nanosensors. Nat. Mater. 2008, 7, 442-453.

(2) Willets, K. A.; Van Duyne, R.P. Localized surface plasmon resonance spectroscopy and sensing. Annu. Rev. Phys. Chem. 2007, 58, 267-297.

(3) Halas, N. J.; Lal, S.; Chang, W-S.; Link, S.; Nordlander, P. Plasmons in strongly coupled metallic nanostructures. Chem. Rev. 2011, 111, 3913-3961.

(4) Giannini, V.; Fernández-Domínguez, A. I.; Heck, S. C.; Maier, S. A. Plasmonic nanoantennas: fundamentals and their use in controlling the radiative properties of nanoemitters. Chem. Rev. 2011, 111, 3888-3912.

(5) Chu, Y.; Banaee, M. G.; Crozier, K. B. Double-resonance plasmon substrates for surface-enhanced Raman scattering with enhancement at excitation and Stokes frequencies. ACS Nano 2010, 4 (5), 2804-2810.

(6) McFarland, A. D.; Young, M. A.; Dieringer, J. A.; Van Duyne, R. P. Wavelengthscanned surface-enhanced Raman excitation spectroscopy. J. Phys. Chem. B 2005, 109, 11279-11285.

(7) Ye, J.; Wen, F.; Sobhani, H.; Britt Lassiter, J.; Van Dorpe, P.; Nordlander, P.; Halas, N.J. Plasmonic Nanoclusters: Near Field Properties of the Fano Resonance Interrogated with SERS. Nano Lett. 2012, 12 (3), 1660-1667.

(8) Kasera, S.; Biedermann, F.; Baumberg, J.J.; Scherman, O.A.; Mahajan, S. Quantitative SERS Using the Sequestration of Small Molecules Inside Precise Plasmonic Nanoconstructs. Nano Lett. 2012, 12 (11), 5924-5928.

(9) Gallinet, B.; Siegfried, T.; Sigg, H.; Nordlander, P.; Martin, O.J.F. Plasmonic Radiance: 
Probing Structure at the Angström Scale with Visible Light. Nano Lett. 2013, 13 (2), 497-503.

(10) Siegfried, T.; Ekinci, Y.; Martin, O.J.F.; Sigg, H. Gap Plasmons and Near-Field Enhancement in Closely Packed Sub- 10 nm Gap Resonators. Nano Lett. 2013, 13 (11), $5449-5453$.

(11) Li, J.; Chen, C.; Jans, H.; Xiumei, X.; Verellen, N.; Vos, I.; Okumura, Y.; Moshchalkov, V.V.; Lagae, L.; Van Dorpe, P. 300 mm Wafer-level, ultra-dense arrays of Au-capped nanopillars with sub-10 nm gaps as reliable SERS substrates. Nanoscale 2014, 6, 1239112396.

(12) Seok, T. J.; Jamshidi, A.; Eggleston, M.; Wu, M.C. Mass-producible and efficient optical antennas with CMOS-fabricated nanometer-scale gap. Opt. Express 2013, 21 (14), 16561-16569.

(13) Dhakal, A.; Subramanian, A.Z.; Wuytens, P.; Peyskens, F.; Le Thomas, N.; Baets, R. Evanescent excitation and collection of spontaneous Raman spectra using silicon nitride nanophotonic waveguides. Opt. Lett. 2014, 39 (13), 4025-4028.

(14) Peyskens, F.; Subramanian, A.Z.; Neutens, P.; Dhakal, A.; Van Dorpe, P.; Le Thomas, N.; Baets, R. Bright and dark plasmon resonances of nanoplasmonic antennas evanescently coupled with a silicon nitride waveguide. Opt. Express 2015, 23 (3), 3088-3101.

(15) Arnaud, L.; Bruyant, A.; Renault, M.; Hadjar, Y.; Salas-Montiel, R.; Apuzzo, A.; Lérondel, G.; Morand, A.; Benech, P.; Le Coarer, E.; Blaize, S. Waveguide-coupled nanowire as an optical antenna. J. Opt. Soc. Am. A 2013, 30 (11), 2347-2355.

(16) Arango, F. B.; Kwadrin, A.; Koenderink, A. F. Plasmonic Antennas Hybridized with Dielectric Waveguides. ACS Nano 2012, 6 (11), 10156-10167. 
(17) Février, M.; Gogol, P.; Aassime, A.; Mégy, R.; Delacour, C.; Chelnokov, A.; Apuzzo, A.; Blaize, S.; Lourtioz, J-M.; Dagens, B. Giant Coupling Effect between Metal Nanoparticle Chain and Optical Waveguide. Nano Lett. 2012, 12, 1032-1037.

(18) Février, M.; Gogol, P.; Lourtioz, J-M.; Dagens, B. Metallic nanoparticle chains on dielectric waveguides: coupled and uncoupled situations compared. Opt. Express 2013, 21 (21), 24504-24513.

(19) Février, M.; Gogol, P.; Barbillon, G.; Aassime, A.; Mégy, R.; Bartenlian, B.; Lourtioz, J-M.; Dagens, B. Integration of short gold nanoparticles chain on SOI waveguide toward compact integrated bio-sensors. Opt. Express 2012, 20 (16), 17402-17410.

(20) Chamanzar, M.; Xia, Z.; Yegnanarayanan, S.; Adibi, A. Hybrid integrated plasmonicphotonic waveguides for on-chip localized surface plasmon resonance (LSPR) sensing and spectroscopy. Opt. Express 2013, 21 (26), 32086-32098.

(21) Lin, S.; Zhu, W.; Jin, Y.; Crozier, K.B. Surface-Enhanced Raman Scattering with Ag Nanoparticles Optically Trapped by a Photonic Crystal Cavity. Nano Lett. 2013, 13 (2), 559-563.

(22) Kong, L.; Lee, C.; Earhart, C.M.; Cordovez, B.; Chan, J.W. A nanotweezer system for evanescent wave excited surface enhanced Raman spectroscopy (SERS) of single nanoparticles. Opt. Express 2015, 23 (5), 6793-6802.

(23) Measor, P.; Seballos, L.; Yin, D.; Zhang, J.Z.; Lunt, E.J.; Hawkins, A.R.; Schmidt, H. On-chip surface-enhanced Raman scattering detection using integrated liquid-core waveguides. Appl. Phys. Lett. 2007, 90, 211107-1-211107-3.

(24) Mahmoud, M.A. Surface-Enhanced Raman Spectroscopy of Double-Shell Hollow Nanoparticles: Electromagnetic and Chemical Enhancements. Langmuir 2013, 29, 6253-6261. 
(25) Martens, D.; Subramanian, A.; Pathak, S.; Vanslembrouck, M.; Bienstman, P.; Bogaerts, W.; Baets, R. Compact silicon nitride arrayed waveguide gratings for very nearinfrared wavelengths. IEEE Photon. Technol. Lett. 2015, 27(2), 137-140.

(26) Pelton, M. Modified spontaneous emission in nanophotonic structures. Nature Photon. 2015, 9, 427-435.

(27) Tame, M.S.; McEnery, K.R.; Özdemir, S.K.; Lee, J.; Maier, S.A.; Kim, M.S. Quantum plasmonics. Nature Phys. 2013, 9, 329-340.

(28) Johnson, P.B.; Christy, R.W. Optical Constants of the Noble Metals. Phys. Rev. B 1972, 6 (12), 4370-4379.

(29) Haynes, W. M., Eds. CRC Handbook of Chemistry and Physics 95th Edition (Internet Version 2015); CRC/Taylor and Francis, 2015.

(30) Thomas, M.; Mühlig, S.; Deckert-Gaudig, T.; Rockstuhl, C.; Deckert, V.; Marquetand, P. Distinguishing chemical and electromagnetic enhancement in surface-enhanced Raman spectra: The case of para-nitrothiophenol. J. Raman Spectrosc. 2013, 44, 14971505.

\section{Acknowledgement}

The authors acknowledge Josine Loo (imec) for performing the e-beam lithography, Liesbet Van Landschoot for making the SEM images, Ananth Subramanian for useful discussions and Stephane Clemmen for providing feedback on the manuscript. This research was funded by the ERC grant InSpectra. F.P acknowledges support from the Bijzonder Onderzoeksfonds (BOF) fellowship of Ghent University. 


\section{Graphical TOC Entry}

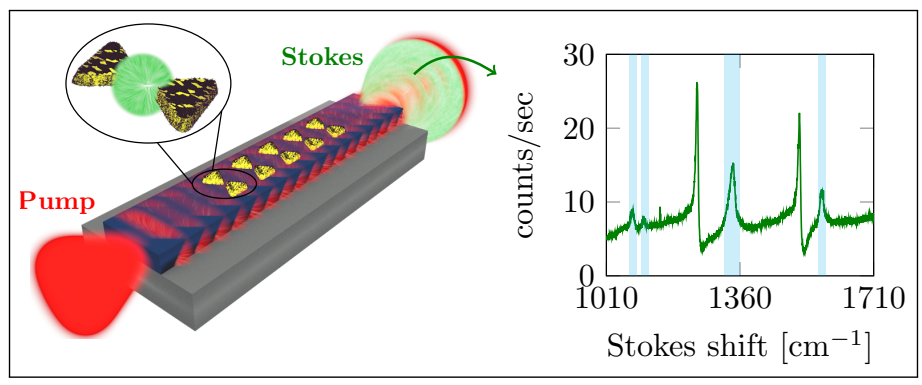

